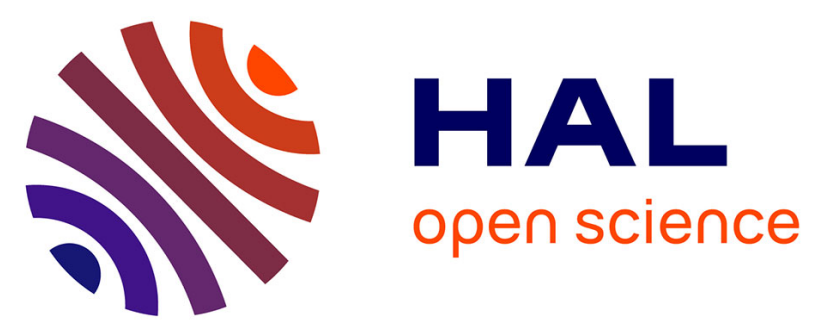

\title{
How bio-inspiration enhances the potential of phononic crystals and metamaterials
}

\author{
M. Miniaci, A. Bergamini, F. Bosia, A. S Gliozzi, N. M Pugno
}

\section{To cite this version:}

M. Miniaci, A. Bergamini, F. Bosia, A. S Gliozzi, N. M Pugno. How bio-inspiration enhances the potential of phononic crystals and metamaterials: [Invited]. 2021 Fifteenth International Congress on Artificial Materials for Novel Wave Phenomena (Metamaterials), Sep 2021, NYC, United States. pp.269-271, 10.1109/metamaterials52332.2021.9577112 . hal-03440823

\section{HAL Id: hal-03440823 \\ https://hal.science/hal-03440823}

Submitted on 22 Nov 2021

HAL is a multi-disciplinary open access archive for the deposit and dissemination of scientific research documents, whether they are published or not. The documents may come from teaching and research institutions in France or abroad, or from public or private research centers.
L'archive ouverte pluridisciplinaire HAL, est destinée au dépôt et à la diffusion de documents scientifiques de niveau recherche, publiés ou non, émanant des établissements d'enseignement et de recherche français ou étrangers, des laboratoires publics ou privés. 


\title{
How bio-inspiration enhances the potential of phononic crystals and metamaterials
}

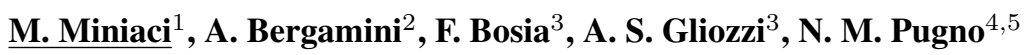 \\ ${ }^{1}$ CNRS, Centrale Lille, ISEN, Univ. Lille, Univ. Valenciennes, UMR 8520 - IEMN, F-59000 Lille, France \\ ${ }^{2}$ Empa, Laboratory for Acoustics/Noise Control, berlandstrasse 129, 8600, Dbendorf, Switzerland \\ ${ }^{3}$ Politecnico di Torino, Department of Applied Science and Technology, Corso Duca degli Abruzzi 24, \\ 10124, Torino, Italy \\ ${ }^{4}$ University of Trento, Laboratory of Bio-Inspired, Bionic, Nano, Meta Materials \& Mechanics, \\ Department of Civil, Environmental and Mechanical Engineering, Via Mesiano77, 38123 Trento, Italy \\ ${ }^{5}$ Queen Mary University of London, School of Engineering \& Materials Science, Mile End Road, London \\ E1 4NS, UK \\ email of the presenter: marco.miniaci@gmail.com
}

\begin{abstract}
Nature has always represented a fundamental source of inspiration to solve mankind's scientific challenges and engineering tasks. For instance, it has been shown that a hierarchical organization over multiple length scales allows enhanced quasi-static mechanical properties, while the relative orientation of adjacent chiral centers strongly affects the physical properties of a polymer, and internal heterogeneous architectures may result in shape changing systems, to cite a few examples. In this paper, we discuss how bio-inspiration may be used to enhance the potential of phononic crystals and acoustic metamaterials.
\end{abstract}

\section{INTRODUCTION AND METHODS}

Phononic crystals and acoustic metamaterials can be considered as composites with ad-hoc designed architectures made of periodic, quasi-periodic or even randomly disposed building blocks (unit cells) exhibiting extraordinary dynamic properties, such as frequency-dependent directionality and band gap (BG) behavior [1]. Since their introduction a few decades ago, researchers have tried to explore more and more configurations capable of (i) achieving low and broadband frequency effects (without recurring to unpractical increases of the unit cell size or stiffness decrease) and (ii) practical solutions to exhibit a tunable frequency response, once the structures are fabricated.

On the other hand, Nature has always represented a formidable source of inspiration to solve mankind's scientific challenges and engineering tasks. For instance, it has been shown that a hierarchical organization over multiple length scales allows enhanced quasi-static mechanical properties, while the relative orientation of adjacent chiral centers strongly affects the physical properties of a polymer, and internal heterogeneous architectures may result in shape changing systems, to cite a few examples [2]. In this paper, we discuss how bio-inspiration may be used to enhance the potential of phononic crystals and acoustic metamaterials [3]. Specifically, a comparison of the dynamic behavior of conventional and bio-inspired phononic crystals / metamaterials is performed through the evaluation of the corresponding dispersion diagrams and / or transmission properties.

From the modeling point of view, we consider small-amplitude waves. In the case of a linear elastic medium, in the absence of external forces, the wave field is described by standard wave equations for two-dimensional displacements, and the analysis is restricted to harmonic waves. Due to the structural periodicity of the phononic crystals / metamaterials, the displacements are also periodic in space and can be thus represented using the Floquet-Bloch expansion theorem. This allows to restrict the analysis of an infinite periodic medium to that of a representative unit cell with Bloch periodic conditions at the unit cell boundaries. 

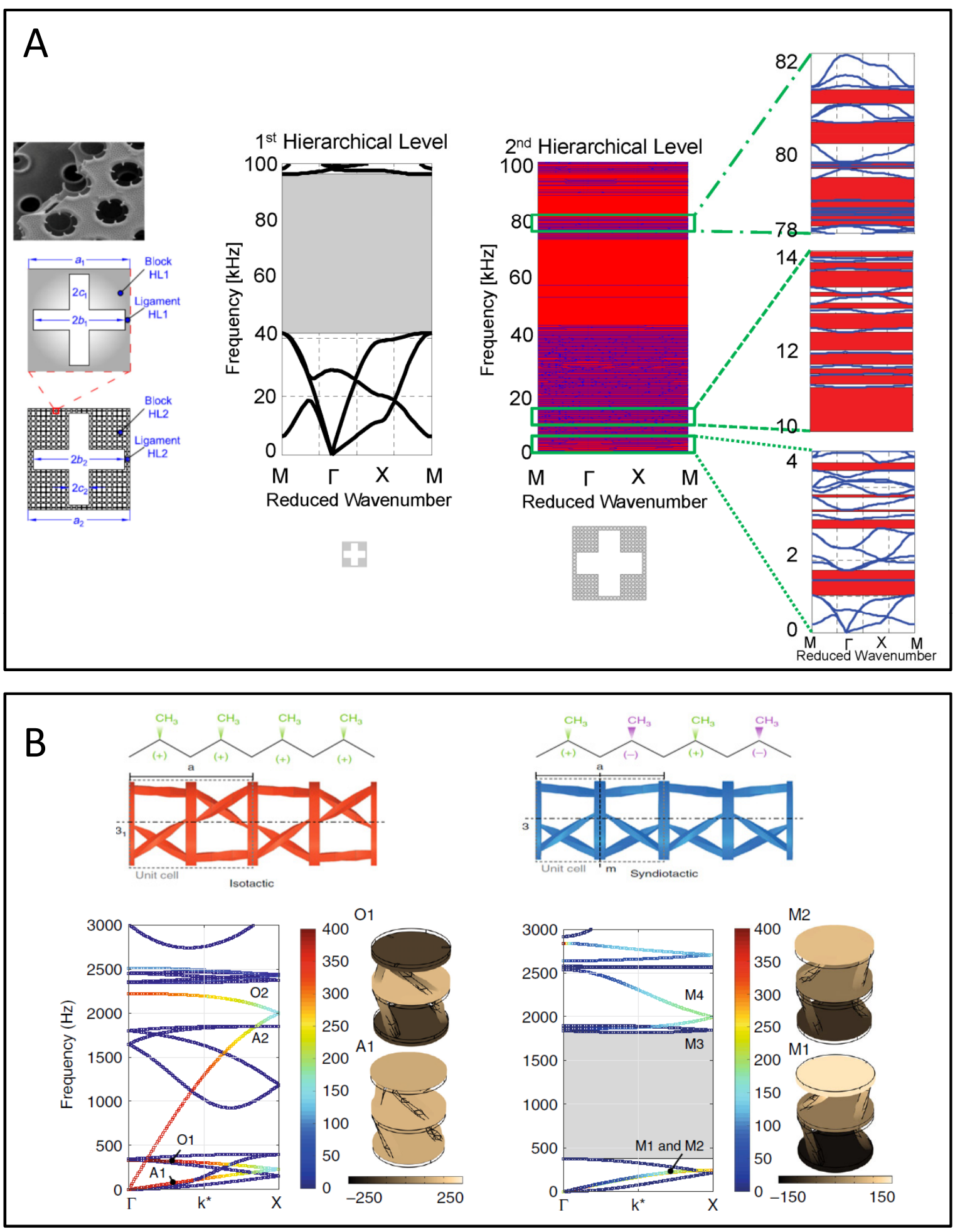

Fig. 1: Figure 1. (A) Hierarchical structures found in natural diatom cell walls and the corresponding bio-inspired hierarchical metamaterial unit cells, along with their dispersion diagrams. The hierarchical organization allows to preserve the BG originating from a smaller constitutive geometry and opening frequency band gaps at lower frequencies. (B) Schematic representation of the concept of tacticity inspiring the design of two periodic diadic arrays of translational-rotational resonators in isotactic and syndiotactic configuration, along with their band diagrams and mode shapes. A large BG between 374 and $1816 \mathrm{~Hz}$ in the syndiotactic arrangement is visible. 


\section{StRUCTURAL HIERARCHY}

Structural hierarchy is here understood in the sense that a representative metamaterial unit cell comprises multiple arrangements of inhomogeneities at various size scales. If the same arrangement occurs at every scale, the pattern is called self-similar. We consider single-phase metamaterials formed by self-similar unit cells with different hierarchical levels, as schematically shown in Fig. 1A.

The dispersion diagrams for the hierarchical cross-like metamaterial with regular and external hierarchical organization is shown in Figures 1A. It clearly appears that the wide band gap ranging from approximately $42 \mathrm{kHz}$ to $96 \mathrm{kHz}$ is almost preserved. Pass bands located inside the latter band gap are represented by mostly flat lines corresponding to localized modes. These modes do not couple with the external field in realistic structures, which always have a certain level of energy dissipation. Also, the introduction of hierarchy allows to shift the band gap corresponding to the ordinary unit cell to an approximately 3 times lower frequency. Several other band gaps appear at the band gap frequencies of the regular structure [4]. The BG shift can be seen as an alteration of the ratio stiffness over mass (decreasing the first more than the second), where the unit cell assembly strategy has been driven by a simple translation of the unit cell.

\section{TACTICITY}

All the classical amino acids present in nature are chiral (except glycine). A molecule is called chiral if it cannot be superimposed on its mirror image by any combination of rotations and translations. While the concept of chirality itself has been largely exploited in the field of phononic crystals and acoustic metamaterials in recent years, the effects of the concatenation of such non-centrosymmetric unit cells (tacticity from polymer science), has not been explored, yet. Inspired by this concept, we show its effects on the dynamic behavior of chiral structured periodic media, by contrasting the dispersion properties of iso- and a syndiotactic crystals.

Our numerical results show that the syndiotactic phononic crystal arrangement nucleates low-frequency full bandgaps, while the isotactic variant exhibits wave modes in the same frequency range, responsible for a transmissive behavior (see Fig. 1B). Nonetheless, the two variants are characterized by the same density and quasistatic stiffness [5].

\section{Conclusions}

In conclusion, we have shown that as the dynamic properties of phononic crystals / metamterials can be enhanced by taking inspiration from biology-related concepts. Specifically, we have proved that a hierarchical organization of self-similar unit cells may lead to multiple BG opening at different frequency scales, as well as to the lowering of the existing ones (whose frequency width is partially preserved). Similarly, concatenation of noncentrosymmetric unit cells leads to a BG frequency lowering, keeping the overall mass and stiffness unchanged.

\section{ACKNOWLEDGEMENT}

This project has received funding from the European Union Horizon 2020 research and innovation programme under grant agreement No. 863179.

\section{REFERENCES}

[1] M. I. Hussein, M. J. Leamy and M. Ruzzene, "Dynamics of Phononic Materials and Structures: Historical Origins, Recent Progress, and Future Outlook," Applied Mechanics Reviews, vol. 66(4), p. 040802, 2014.

[2] M. Meyers, P. Chen, A. Lin and Y. Seki, "Biological materials: Structure and mechanical properties," Progress in Materials Science, vol. 53, p. 1-206, 2008.

[3] M. Miniaci, A. Krushynska, A. B. Movchan, F. Bosia, and N. M. Pugno, "Spider web-inspired acoustic metamaterials," Applied Physics Letters, vol. 109, p. 071905, 2016.

[4] M. Miniaci, A. Krushynska, A. S. Gliozzi, N. Kherraz, F. Bosia, and N. M. Pugno, "Design and Fabrication of Bioinspired Hierarchical Dissipative Elastic Metamaterials,” Physical Review Applied, vol. 10, p. 024012, 2018.

[5] A. Bergamini, M. Miniaci, T. Delpero, D. Tallarico, B. Van Damme, G. Hannema, I Leibacher, A. Zemp, "Tacticity in chiral phononic crystals," Nature Communications, vol. 10, p. 4525, 2019. 